\title{
COMMENT
}

\section{Global Pediatric Research Investigator: Dr. Nadia A. Sam-Agudu}

\author{
Nadia A. Sam-Agudu (D) ${ }^{1,2}$ \\ Pediatric Research (2021) 89:1062; https://doi.org/10.1038/s41390-020-01339-9
}

I was born in Accra, Ghana, and after completing Advanced-Level secondary school, I left to study at the University of Virginia, Charlottesville, USA. I graduated with a BA in Interdisciplinary African Studies, as I had plans to pursue a medical career involving African children.

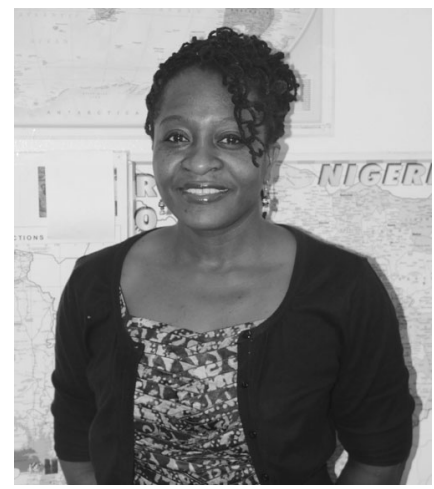

I initially had little interest in research; however, it was a requirement for all students at Mayo Medical School, Rochester, Minnesota. For my project and a first taste of research, I returned to Ghana to study pediatric malaria immunology at the Noguchi Memorial Institute for Medical Research.

After graduating medical school in 2002, I completed a pediatric residency and an Infectious Diseases fellowship at the University of Minnesota in Minneapolis. There, I met Dr. Chandy John, who became my fellowship mentor. Dr. John's research focused on pediatric malaria immunology in East Africa, which was a great fit for me. As a budding clinician-scientist, I flourished under Dr. John's excellent, supportive mentorship and steadily developed my translational research skills. I have always loved writing, and transitioned easily from creative to scientific writing.

In 2010, I joined the Epidemiology and Prevention Division of the Institute of Human Virology, University of Maryland School of Medicine, then led by Dr. William Blattner. However, I have been based full-time in Nigeria as Senior Technical Advisor for Pediatric/Adolescent HIV at the Institute of Human Virology Nigeria, Abuja. My research leverages on implementation science methods to sustainably improve disease prevention and treatment outcomes for children, especially in HIV, malaria, tuberculosis, and now, COVID-19. As a Principal Investigator, my grants include prevention of mother-to-child transmission of HIV and adolescent HIV studies funded by the World Health Organization, the American Academy of Pediatrics, and the NIH. I have concentrated my work in West and Central Africa, which lags behind the rest of Africa in child health indices and research evidence generated. I am an active member of the Nigeria Implementation Science Alliance (NISA), and I lead the Central and West Africa Implementation Science Alliance (CAWISA).

Clinical knowledge is important in generating relevant research questions and in applying evidence to practice. I, therefore, appreciate pediatricians Dr. Patricia Ferrieri, Dr. Laura Hoyt, Dr. Kiran Belani, Dr. Stacene Maroushek, and Dr. Andrew Kiragu for their superb clinical mentorship during my training.

To up-and-coming colleagues in pediatric research:

- Research what moves you.

- Find mentors who value you and your goals, and be an industrious mentee.

- Find long-term collaborators. They will exponentially increase your research productivity.

- When collaborating, be responsive to communication, give value, and be pleasant to work with.

- Have fun!

\section{ACKNOWLEDGEMENTS}

NASA's work is funded by the NIH/National Institute of Child Health and Human Development (NICHD), award \#R01HD089866, and by an award from the NIH Fogarty International Center via the Adolescent HIV Prevention and Treatment Implementation Science Alliance (AHISA), for the Central and West Africa Implementation Science Alliance (CAWISA)

\section{ADDITIONAL INFORMATION}

Competing interests: The author declares no competing interests.

Publisher's note Springer Nature remains neutral with regard to jurisdictional claims in published maps and institutional affiliations.

${ }^{1}$ Institute of Human Virology and Department of Pediatrics, University of Maryland School of Medicine, Baltimore, MD, USA and ${ }^{2}$ Pediatric/Adolescent HIV Unit and International Research Center of Excellence, Institute of Human Virology Nigeria, Abuja, Nigeria

Correspondence: Nadia A. Sam-Agudu (nsam-agudu@ihv.umaryland.edu)

Received: 16 September 2020 Revised: 26 September 2020 Accepted: 4 December 2020

Published online: 14 January 2021 